Research paper

\title{
Enhanced baseline activity in the left ventromedial putamen predicts individual treatment response in drug-naive, first-episode schizophrenia: Results from two independent study samples
}

\author{
Huabing Li ${ }^{\mathrm{a}}$, Wenbin Guo ${ }^{\mathrm{b}, \mathrm{c}, *}$, Feng Liu ${ }^{\mathrm{d}}$, Jindong Chen ${ }^{\mathrm{b}, \mathrm{c}}$, Qinji Su ${ }^{\mathrm{e}}$, Zhikun Zhang ${ }^{\mathrm{e}}$, \\ Xiaoduo Fan ${ }^{\mathrm{f}, * *}$, Jingping Zhao ${ }^{\mathrm{b}, \mathrm{c}}$ \\ a Department of Radiology, The Second Xiangya Hospital of Central South University, Changsha, Hunan 410011, China \\ b Department of Psychiatry, The Second Xiangya Hospital of Central South University, Changsha, Hunan 410011, China \\ c National Clinical Research Center on Mental Disorders, Changsha, Hunan 410011, China \\ d Department of Radiology, Tianjin Medical University General Hospital, Tianjin 300000, China \\ e Mental Health Center, The Second Affiliated Hospital of Guangxi Medical University, Nanning, Guangxi 530007, China \\ ${ }^{\mathrm{f}}$ University of Massachusetts Medical School, UMass Memorial Medical Center, One Biotech, Suite 100, 365 Plantation Street, Worcester, MA 01605, United States
}

\section{A R T I C L E I N F O}

\section{Article history:}

Received 1 April 2019

Received in revised form 20 June 2019

Accepted 8 July 2019

Available online $\mathrm{xxxx}$

\section{Keywords:}

Schizophrenia

Olanzapine

Fractional amplitude of low frequency fluctuation

Pattern classification

\begin{abstract}
S U M M A R Y
Background: Antipsychotic medications are the common treatment for schizophrenia. However, reliable biomarkers that can predict individual treatment response are still lacking. The present study aimed to examine whether baseline putamen activity can predict individual treatment response in schizophrenia.

Methods: Two independent samples of patients with drug-naive, first-episode schizophrenia (32 patients in sample 1 and 44 in sample 2) and matched healthy controls underwent resting-state functional magnetic resonance imaging (fMRI) at baseline. Patients were treated with olanzapine for 8 weeks; symptom severity was assessed using the Positive and Negative Syndrome Scale (PANSS) at baseline and week 8. Fractional amplitude of low frequency fluctuation (fALFF) and pattern classification techniques were used to analyze the data.

Findings: Univariate analysis shows an elevated pre-treatment fALFF in the left ventromedial putamen in both patient samples compared to healthy controls ( $p$ 's $<0.001$ ). The support vector regression (SVR) analysis suggests a positive relationship between baseline pre-treatment fALFF in the left ventromedial putamen and improvement in positive symptom at week 8 in each patient group using a cross-validated method $(r=0.452, p=.002 ; r=$ $0.511, p=.003$, respectively).

Interpretation: Our study suggests that elevated pre-treatment mean fALFF in the left ventromedial putamen may predict individual therapeutic response to olanzapine treatment in drug-naive, first-episode patients with schizophrenia. Future studies are needed to confirm whether this finding is generalizable to patients with schizophrenia treated with other antipsychotic medications.

Fund: The National Key R\&D Program of China and the National Natural Science Foundation of China.
\end{abstract}

(c) 2019 The Authors. Published by Elsevier B.V. This is an open access article under the CC BY-NC-ND license (http:// creativecommons.org/licenses/by-nc-nd/4.0/).

\section{Introduction}

Putamen, a key component of the striatum, receives afferent information from cortical regions (i.e., the prefrontal cortex), and sends information back to cortical regions via thalamus [1]. Through the cortico-striato-thalamo-cortical circuit, putamen plays a critical role in

\footnotetext{
* Corresponding author at: Department of Psychiatry, The Second Xiangya Hospital of Central South University, Changsha, Hunan 410011, China.

** Corresponding author.

E-mail addresses: guowenbin76@csu.edu.cn (W. Guo), xiaoduo.fan@umassmed.edu (X. Fan).
}

the modulation and integration of motor, emotion, motivation, and cognitive function [2-4].

Structural abnormalities and dysfunction of putamen might be involved in schizophrenia $[5,6]$. For example, increased putamen size was associated with decreased positive symptoms of schizophrenia after six weeks of treatment [7]. In contrast, putamen shrinkage was found in patients with schizophrenia who had never been exposed to antipsychotic medications [8]. Reduced putamen size was observed in patients who discontinued treatment, whereas increased putamen size was found in patients with continued antipsychotic treatment including olanzapine, quetiapine, and risperidone [9]. In addition, elevated metabolism in putamen has been reported in patients with 


\section{Research in context \\ Evidence before this study}

We searched PubMed from the start of the database until April 1 2019, for putamen activity to predict treatment response in schizophrenia, with the search terms "schizophrenia", "putamen", and "treatment response [Title]", and "English" [Language]. We found two published articles before we initiated the study. In these studies, gray matter volume (GMV) was used to predict treatment response in schizophrenia. One study demonstrated that the caudate GMV changes after treatment were able to predict treatment response after 6 weeks of risperidone treatment. The other study reported that an increased putamen GMV was associated with decreased positive symptoms of schizophrenia after six weeks of antipsychotic treatment. Several important confounding and/or limiting factors, such as long illness duration, previous exposure to antipsychotic medication, a gross brain atlas used, prediction at the group level rather than individual patient level, exploration of post-treatment predictors rather than pretreatment predictors, and lack of cross-validation of the predictive model, may have complicated the findings from previous studies.

\section{Added value of this study}

The present study intended to answer a clinically relevant key question: can baseline pre-treatment putamen activity serve as a biomarker to predict therapeutic response in patients with schizophrenia?

Two independent samples of patients with drug-naive, firstepisode schizophrenia (32 patients in sample 1 and 44 in sample 2) and matched healthy controls underwent resting-state functional magnetic resonance imaging ( $\mathrm{fMRI}$ ) at baseline. The present study aimed to examine the role of baseline pre-treatment putamen in predicting therapeutic response to olanzapine treatment in two independent samples of patients with drug-naive, firstepisode schizophrenia using a combination of hypothesis-driven and data-driven approaches. The support vector regression (SVR) analysis suggests that baseline levels of fALFF in the left ventromedial putamen can predict improvement in positive symptoms after 8 weeks of olanzapine treatment.

\section{Implications of all the available evidence}

First, the left ventromedial putamen activity may predict individual therapeutic response to olanzapine treatment in drug-naive, firstepisode patients with schizophrenia. Second, the fact that the findings were consistent across two independent study samples cross-validates the predictive value of the left ventromedial putamen activity for antipsychotic treatment response. Finally, such a pre-treatment predictor, if further confirmed, is helpful for psychiatrists to make an informed decision regarding the choice of antipsychotic medications when initiating treatment.

chronic schizophrenia who received antipsychotic treatment [10]. Furthermore, different antipsychotic drugs have different effects on putamen size. For example, exposure to quetiapine might result in reduced putamen density [11], whereas exposure to risperidone could lead to putamen enlargement [12].

Given the potential role of putamen in schizophrenia and the fact that antipsychotic treatment is associated with both structural and functional changes in putamen, a clinically relevant key question would be whether baseline pre-treatment putamen activity can serve as a biomarker to predict therapeutic response in patients with schizophrenia. To answer this question, several important confounding and/or limiting factors, which often complicated the findings from previous studies, must be addressed. For example, chronic and medicated patients were enrolled in many previous studies; it has been well established that both disease duration and exposure to antipsychotic treatment may have neurotoxic effect on the brain [13-18]. In addition, previous studies often included patients who were on different antipsychotic agents, which may have differential effects on the brain $[19,20]$. Further, the imaging predictors explored previously were brain structural and functional alterations after antipsychotic therapy [7,21]. There is still lack of studies focusing on pre-treatment imaging predictors, which are helpful for psychiatrists to make an informed decision regarding the choice of antipsychotic medications before initiating a treatment.

Many previous brain atlases are based on ex vivo histological atlases, and lack of fine-defined parcellations to provide in vivo information [22]. Recently, a connectivity-based brainnetome atlas became available to offer a fine-grained, cross-validated brain atlas [22]. According to this new atlas, putamen is divided into four subregions: left/right ventromedial putamen and left/right dorsolateral putamen. Hence, regional specific alterations of putamen can be explored in detail.

Most previous studies used univariate statistics to predict treatment outcome at the group level [23], which is of little utility in everyday clinical practice when psychiatrists need to make treatment decision at the individual patient level. Pattern classification techniques, such as support vector machine (SVM) and support vector regression (SVR), allow predictions to be made at the individual level $[24,25]$. Such techniques have been used to discriminate patients with adolescent-onset schizophrenia from healthy controls [26-28], and to predict individual response to electroconvulsive treatment in major depressive disorder [29].

In most of previous clinical prediction studies, there was a lack of validation of the predictive model in an independent patient sample. Therefore, it remains unclear, to what extent, that the proposed predictive model can be replicated and validated in patients other than the single study sample.

In the present study, we recruited two independent samples of drugnaive, first-episode patients with schizophrenia from two different geographic locations in China. Functional magnetic resonance imaging (fMRI) scans were obtained at baseline (prior to the initiation of antipsychotic treatment). Symptom severity was assessed at baseline and after receiving 8 weeks of olanzapine treatment. Using the fractional amplitude of low frequency fluctuation (fALFF) method and pattern classification techniques including SVR, the present study was to examine the activity of putamen in drug-naive, first-episode patients with schizophrenia and its role in predicting individual therapeutic response to olanzapine treatment. Hypothesis-driven and data-driven approaches were used in the present study. Based on the abovementioned studies, increased regional activity in putamen was expected to predict individual therapeutic response to olanzapine treatment (hypothesisdriven). The putamen was divided into four subregions: left/right ventromedial putamen and left/right dorsolateral putamen. The datadriven approach was used to determine which subregion with abnormal activity in the two study samples was used to predict individual therapeutic response to olanzapine treatment.

\section{Materials and methods}

\subsection{Subjects}

Sample 1 included 34 drug-naive, first-episode patients with schizophrenia and 34 healthy controls. Patients were recruited from the Department of Psychiatry, the Second Xiangya Hospital of Central South University in Hunan Province, China; healthy controls were recruited from the local community. Sample 2 included 46 drug-naive, first-episode patients with schizophrenia and 46 healthy controls. Patients were recruited from the Mental Health Center, the Second 
Affiliated Hospital of Guangxi Medical University in Guangxi Province, China; healthy controls were recruited from the local community.

Both patient and healthy controls were Han Chinese with the age range 18-50 years old and right-handed, and had $>6$ years of formal education. Patients were diagnosed with schizophrenia according to the Diagnostic and Statistical Manual of Mental Disorders-IV (DSM-IV). They were never treated with antipsychotic medications or other psychotropic agents. The diagnosis of schizophrenia was further determined by two research psychiatrists (W.G. and Z.Z.) using the Structural Clinical Interview for DSM-IV (SCID), patient version [30]. All patients had a Positive and Negative Syndrome Scale (PANSS) total score of $>70$ at baseline. Exclusion criteria included history of alcohol or other substance use, history of brain injury, or any ongoing significant medical conditions.

Healthy controls were recruited through advertisement. A complete medical history, physical examination, and routine laboratory tests were obtained for all healthy controls to rule out possible medical conditions; in addition, the same research psychiatrists (W.G. and Z.Z.) conducted a structured clinical interview using the SCID, non-patient version [30] to rule out any psychiatric conditions. None of them had a history of any psychiatric conditions, alcohol or substance use; further, those who had a first-degree relative diagnosed with psychiatric disorders were also excluded from the study.

The study was reviewed and approved by the ethics committees of the Second Xiangya Hospital of Central South University and the Second Affiliated Hospital of Guangxi Medical University. A written informed consent was obtained from each subject.

\subsection{Procedure}

After the screening visit, those individuals who fit the inclusion and exclusion criteria were enrolled in the study. All subjects went through the brain MRI imaging scan and symptom severity was assessed using the PANSS at baseline. Then patient subjects received olanzapine (10$30 \mathrm{mg} /$ day as clinically appropriate and tolerated) for 8 weeks. The clinical symptoms were assessed again at week 8 using the PANSS.

\subsection{Data acquisition and preprocessing}

Imagining scans were acquired using 3 T MRI scanners (Siemens Verio, Erlangen, Germany). The data were preprocessed using the DPABI software [31]. The details of data acquisition and preprocessing are provided in eMethods 1 in the Supplement.

\section{4. fALFF}

The fALFF method in our study is similar as used in a previous study [32]. The details for fALFF calculation can be found in the Supplement. After that, mean fALFF values in the subregions (left/right ventromedial putamen and left/right dorsolateral putamen) of the putamen were extracted from each subject using masks (Fig. 1) from the human brainnetome atlas (http://atlas.brainnetome.org) [22].

\subsection{Statistical analyses}

Analysis of variance (ANOVA), independent $t$-tests, and Chi-square tests were employed to analyze demographic and clinical data across groups as appropriate.

Analyses of covariance (ANCOVA) with Sample (sample 1, sample 2) and Diagnosis (patients, controls) as between subject factors controlling for potential confounding variables, were conducted to determine the Sample $\times$ Diagnosis interactions for baseline pre-treatment fALFF values in each subregion. Framewise displacement (FD) was calculated for each subject according to a previous study [33]. The mean FD and age served as covariates in the analyses. The significance level was set at $p<.05$.
If the Sample $\times$ Diagnosis interactions were not significant, further post hoc $t$-tests were used to compare group differences (patients versus controls) in baseline pre-treatment fALFF values in each subregion averaging over both samples. The significance level was set at $p<.05$.

To assess treatment outcome, the following formula was used to calculate the reduction ratio (RR) of the PANSS total scores.

$$
\mathrm{RR}=\left(\mathrm{PANSS}_{\text {total_0}}-\mathrm{PANSS}_{\text {total_8w }}\right) / \text { PANSS }_{\text {total_0 }}
$$

PANSS $_{\text {total } \_0}$ refers to the PANSS total scores at baseline (pre-treatment), whereas PANSS total_8w $_{\text {is }}$ the PANSS total scores after 8 weeks of olanzapine treatment. Similar RRs were calculated for the PANSS Positive Symptoms, Negative Symptoms and General Psychopathology subscale scores.

To test whether there were correlations in brain activity between the left and right ventromedial/dorsolateral putamen, Pearson correlation analyses were performed for fALFF values between the left and right ventromedial/dorsolateral putamen within the groups.

Pearson correlation analyses were also conducted between the pretreatment fALFF values and pre-treatment PANSS scores within each patient sample.

\subsection{Pattern classification analyses}

The LIBSVM software (http://www.csie.ntu.edu.tw/ cjlin/libsvm/) was used to perform pattern classification analysis. Once the subregions with abnormal fALFF were identified in both patient samples, mean fALFF values in such subregions were selected to run the SVR analyses (data-driven). For each subregion and each symptom domains (the PANSS total and Positive Symptoms, Negative Symptoms and General Psychopathology subscale scores), SVR was conducted and crossvalidated with one sample as a train set and the other sample as a test set. SVR was used to individually predict symptom improvement as reflected by the RR. The SVR results were validated by a permutation test, which ran 1000 times for each SVR result.

\section{Results}

\subsection{Baseline characteristics of the subjects}

Seven scans ( 2 patients and 1 control from Sample $1 ; 2$ patients and 2 controls from Sample 2) were excluded due to excessive head motion, and one healthy control from Sample 1 withdrew the consent. Hence, the final samples included 32 patients and 32 controls in Sample 1 and 44 patients and 44 controls in Sample 2.

Between Sample 1 and Sample 2, no significant differences were observed gender $\left(x^{2}=1.825, p=.610\right)$, education $(F=2.437, p=.067)$, and $\operatorname{FD}(F=0.424, p=.736)$. However, participants in Sample 1 were older than individuals in Sample $2(F=18.986, p<.001)$. Patients in Sample 1 had shorter illness duration $(t=-8.553, p<.001)$, scored less in the baseline PANSS Positive Symptoms subscale scores $(t=$ $-2.151, p=.035)$, General Psychopathology subscale scores $(t=$ $-7.242, p<.001)$ and total scores $(t=-6.958, p<.001)$. There were no significant differences in dosage of olanzapine $(t=0.253, p=$ $.801)$ and the baseline PANSS Negative Symptoms scores $(t=-1.671$, $p=.099$ ) for patients between the two samples.

Within each study sample, there were no significant differences in age, gender, education level and the mean FD values between patients and healthy controls (p's $>0.250$ ) (Table 1). The PANSS total score and Positive Symptoms, Negative Symptoms and General Psychopathology subscale scores for Sample 1 and Sample 2 are shown in Table 2.

\subsection{Baseline group differences in fALFF}

As shown in Table 3, the patient group in Sample 1 showed higher levels of fALFF in bilateral ventromedial putamen and bilateral 

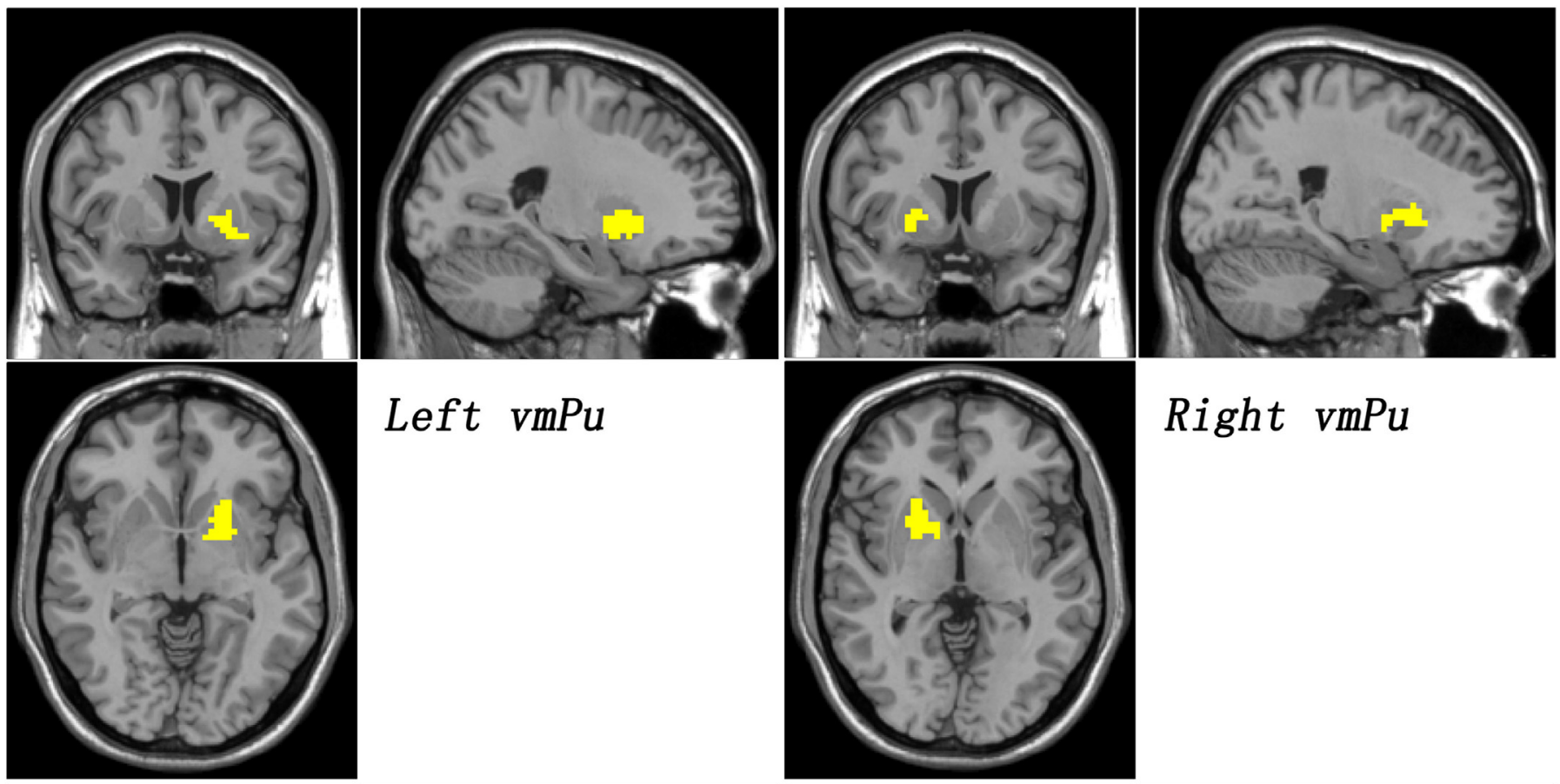

\section{Right $\mathrm{vmPu}$}
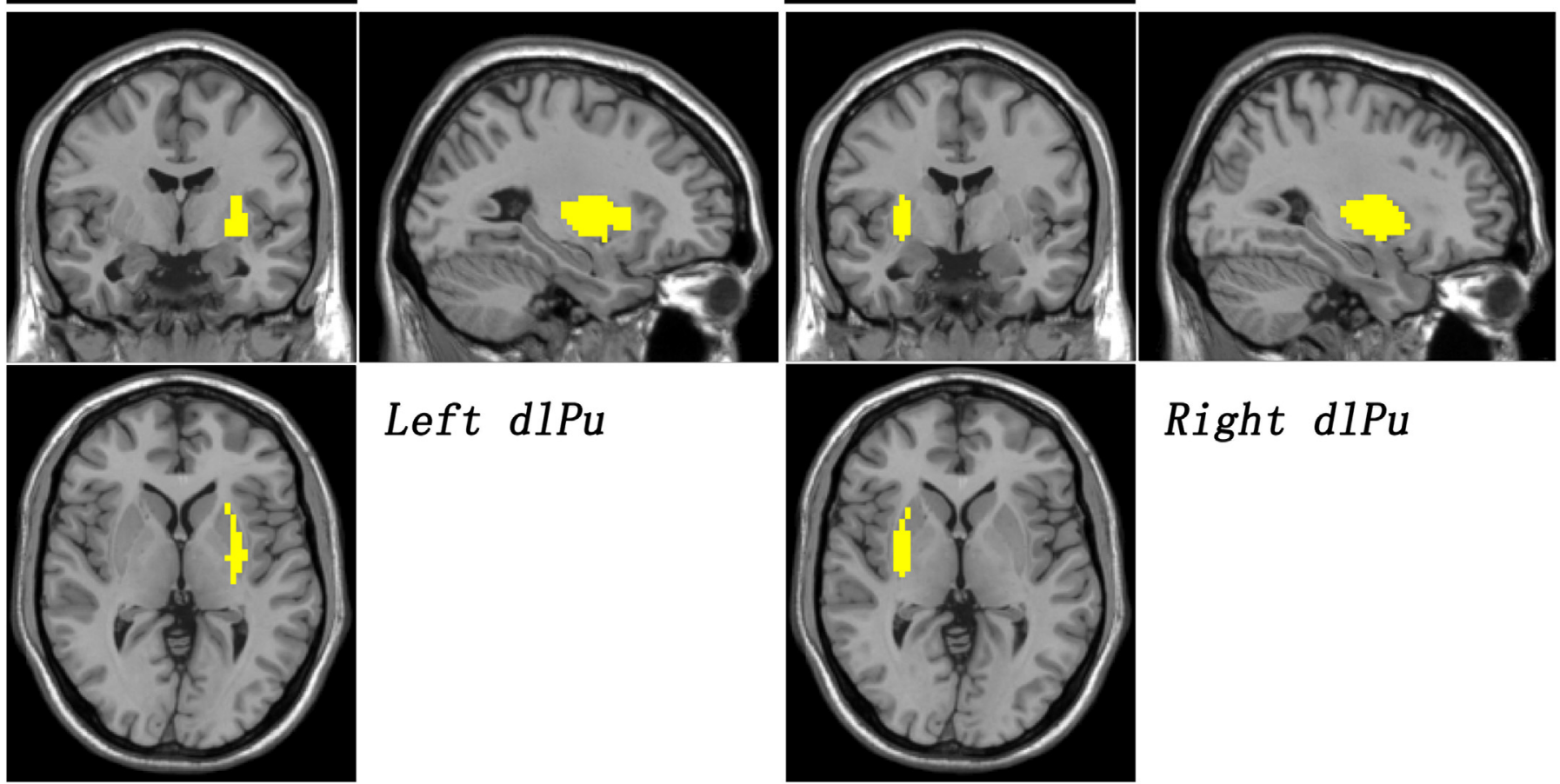

Right $d 1 P u$

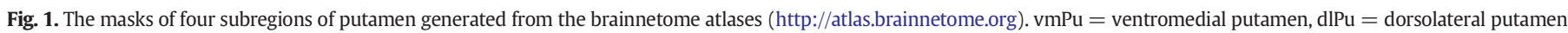

dorsolateral putamen compared to healthy controls. By contrast, the patient group in Sample 2 showed higher levels of fALFF in the left ventromedial putamen compared to the control group.

The ANCOVA controlling for the mean FD and age revealed the Diagnosis Main Effects were significant with $\mathrm{t} / p$ values 5.377/0.000, 2.911/ $0.004,2.866 / 0.005$, and $2.568 / 0.011$ for baseline pre-treatment fALFF in the left ventromedial putamen, left dorsolateral putamen, right ventromedial putamen, and right dorsolateral putamen, respectively.

No significant Sample $\times$ Diagnosis interactions for baseline pretreatment fALFF in 3 subregions. The F/p values were 1.318/0.253, $0.580 / 0.447$, and $1.919 / 0.168$ in the left ventromedial putamen, left dorsolateral putamen, and right dorsolateral putamen, respectively. However, the interaction was close to significant for the right ventromedial putamen with $\mathrm{F} / \mathrm{p}$ values 3.718/0.052.
Based on these observations from the 2 separate study samples, further prediction and pattern classification analyses relating baseline pre-treatment putamen activity to therapeutic response focused on the following 3 subregions: the left ventromedial putamen, left and right dorsolateral putamen.

\subsection{Clinical symptoms after 8 weeks of olanzapine treatment}

As shown in Table 2, after 8 weeks of olanzapine treatment, the patient group in Sample 1 showed significant improvement in the PANSS total score, Positive Symptoms, Negative Symptoms and General Psychopathology subscale scores $(36.50 \pm 2.97,8.91 \pm 1.55,8.97 \pm 1.84$, $18.63 \pm 1.48$ respectively, mean \pm SD), compared to the baseline scores ( $p$ 's $<0.001$ ); the patient group in Sample 2 also showed significant 
Table 1

Baseline demographic and clinical characteristics of the study participants.

\begin{tabular}{llll}
\hline & Patients & Healthy controls & $p$ value \\
\hline Sample 1 & $N=32$ & $\mathrm{~N}=32$ & \\
Gender (male/female) & $16 / 16$ & $17 / 15$ & 0.80 \\
Age (years) & $30.94 \pm 8.25$ & $30.47 \pm 7.69$ & 0.81 \\
Education (years) & $12.13 \pm 3.19$ & $12.41 \pm 2.47$ & 0.70 \\
FD (mm) & $0.03 \pm 0.02$ & $0.04 \pm 0.03$ & 0.26 \\
Illness duration (months) & $8.91 \pm 6.39$ & & \\
Dosage of olanzapine (mg/d) & $18.59 \pm 4.96$ & & \\
Sample 2 & $N=44$ & $\mathrm{~N}=44$ & 0.28 \\
Gender (male/female) & $28 / 16$ & $23 / 21$ & 0.90 \\
Age (years) & $23.45 \pm 4.24$ & $23.55 \pm 2.58$ & 0.69 \\
Education (years) & $11.11 \pm 2.46$ & $11.30 \pm 1.67$ & \\
FD (mm) & $0.03 \pm 0.03$ & $0.03 \pm 0.02$ & \\
Illness duration (months) & $22.34 \pm 7.01$ & & \\
Dosage of olanzapine (mg/d) & $18.30 \pm 5.17$ & & \\
\hline
\end{tabular}

$\mathrm{FD}=$ framewise displacement; values are expressed as mean $\pm \mathrm{SD}$.

improvement in the PANSS total score, Positive Symptoms, Negative Symptoms and General Psychopathology subscale scores (38.70 \pm $7.44,7.50 \pm 1.19,9.50 \pm 2.56,21.70 \pm 5.26$, respectively, mean \pm $\mathrm{SD})$, compared to the baseline scores ( $\mathrm{p}$ 's $<0.001$ ).

\subsection{Correlation results}

As shown in Table S1, significant correlations were observed for pretreatment fALFF values between the left and right ventromedial/dorsolateral putamen within the groups ( $p s<0.001$ ) except the pair of the left and right ventromedial putamen in the controls of Sample $2(p=.073)$.

In addition, there were no significant correlations between pretreatment fALFF values and pre-treatment PANSS total or subscale scores within each patient group.

\subsection{Pattern classification results}

To test whether pre-treatment fALFF in the left ventromedial putamen can predict therapeutic response after 8 weeks of olanzapine treatment, SVR was conducted and cross-validated with one sample as a train set and the other sample as a test set.

When Sample 1 was used as a train set and Sample 2 used as a test set, the SVR analysis showed a significantly positive relationship between pre-treatment levels of fALFF in the left ventromedial putamen and week 8 RR values of the PANSS Positive Symptoms subscale scores $(r=0.452, p=.002$, Fig. 2$)$. The permutation test showed a $p$ value of 0.0020 for this SVR result. No significant correlations were observed between pre-treatment levels of fALFF in the left ventromedial putamen and week 8 RR values of other PANSS scores.

When Sample 2 was used as a train set and Sample 1 used as a test set, the SVR analysis also showed a significantly positive relationship between pre-treatment levels of fALFF in the left ventromedial putamen and week 8 RR values of the PANSS Positive Symptoms subscale scores

Table 2

The PANSS scores for patients at baseline and week 8 .

\begin{tabular}{llll}
\hline PANSS & Baseline & Week 8 & $p$ value \\
\hline Sample 1 $(\mathrm{N}=32)$ & & & \\
Positive symptoms & $20.00 \pm 4.32$ & $8.91 \pm 1.55$ & $<0.001$ \\
Negative symptoms & $20.59 \pm 3.46$ & $8.97 \pm 1.84$ & $<0.001$ \\
General psychopathology & $36.78 \pm 3.68$ & $18.63 \pm 1.48$ & $<0.001$ \\
Total score & $77.38 \pm 5.17$ & $36.50 \pm 2.97$ & $<0.001$ \\
Sample 2 $(\mathrm{N}=44)$ & & & \\
Positive symptoms & $22.48 \pm 5.37$ & $7.50 \pm 1.19$ & $<0.001$ \\
Negative symptoms & $22.50 \pm 6.38$ & $9.50 \pm 2.56$ & $<0.001$ \\
General psychopathology & $45.73 \pm 6.97$ & $21.70 \pm 5.26$ & $<0.001$ \\
Total score & $90.70 \pm 11.17$ & $38.70 \pm 7.44$ & $<0.001$ \\
\hline
\end{tabular}

PANSS $=$ the Positive and Negative Syndrome Scale; values are expressed as mean \pm SD
Table 3

Baseline group comparison in levels of fALFF in different subregions of putamen.

\begin{tabular}{llll}
\hline Subregions of putamen & Patients & Healthy controls & $p$ value \\
\hline Sample 1 & $\mathrm{N}=32$ & $\mathrm{~N}=32$ & \\
Left ventromedial putamen & $0.0231 \pm 0.0747$ & $-0.0477 \pm 0.0650$ & $<0.001$ \\
Left dorsolateral putamen & $-0.0246 \pm 0.0859$ & $-0.0673 \pm 0.0545$ & 0.021 \\
Right ventromedial putamen & $0.0077 \pm 0.0690$ & $-0.0504 \pm 0.0565$ & $<0.001$ \\
Right dorsolateral putamen & $-0.0123 \pm 0.0873$ & $-0.0594 \pm 0.0613$ & 0.015 \\
Sample 2 & $\mathrm{N}=44$ & $\mathrm{~N}=44$ & \\
Left ventromedial putamen & $-0.0004 \pm 0.0636$ & $-0.0464 \pm 0.0566$ & 0.001 \\
Left dorsolateral putamen & $-0.0371 \pm 0.0680$ & $-0.0618 \pm 0.0634$ & 0.082 \\
Right ventromedial putamen & $-0.0529 \pm 0.0781$ & $-0.0662 \pm 0.0647$ & 0.386 \\
Right dorsolateral putamen & $-0.0577 \pm 0.0656$ & $-0.0732 \pm 0.0606$ & 0.252 \\
\hline fALFF $=$ fractional amplitude of low frequency fluctuation; values are expressed as mean \\
\pm SD.
\end{tabular}

( $r=0.511, p=.003$, Fig. 3 ). For this SVR result, the permutation test showed a $p$ value of 0.0143 . There were no significant correlations between pre-treatment levels of fALFF in the left ventromedial putamen and week 8 RR values of other PANSS scores.

Similarly, SVR analyses were conducted for the left/right dorsolateral putamen with one sample as a train set and the other sample as a test set. When Sample 1 was used as a train set and Sample 2 used as a test set, the SVR analysis showed no significant correlations between pre-treatment levels of fALFF in the left/right dorsolateral putamen and week 8 RR values of the PANSS Positive Symptoms subscale scores (left dorsolateral putamen: $r=0.115, p=.458$, eFigure 1 in the Supplement; right dorsolateral putamen: $r=0.042, p=.790$, eFigure 2 in the Supplement). Further, there were also no significant correlations between pre-treatment levels of fALFF in the left/right dorsolateral putamen and week 8 RR values of the PANSS Positive Symptoms subscale scores in patients of Sample 1 when Sample 2 was used as a train set and Sample 1 used as a test set (left dorsolateral putamen: $r=$ $-0.095, p=.604$, eFigure 3 in the Supplement; right dorsolateral putamen: $r=-0.115, p=.532$, eFigure 4 in the Supplement). No significant correlations were observed between pre-treatment levels of fALFF in the left/right dorsolateral putamen and week 8 RR values of other PANSS scores with one sample as a train set and another sample as a test set.

\section{Discussion}

As far as we are aware of, the present study was the first study to examine the role of putamen in predicting therapeutic response to olanzapine treatment in two independent samples of patients with drug-naive, first-episode schizophrenia using a combination of hypothesis-driven and data-driven approaches. The SVR analysis suggests that baseline pre-treatment levels of fALFF in the left ventromedial putamen can predict positive symptom improvement after 8 weeks olanzapine treatment.

Our findings are consistent with a previous study reporting that the volume increase in putamen was associated with positive symptom improvement as measured by the RR of the PANSS Positive Symptoms subscale scores in drug-naive patients with schizophrenia after six weeks of antipsychotic treatment [7]. Our findings are also in line with a previous report that the volume increase in putamen was associated with a better outcome in schizophrenia [23]; in that study, the authors suggested that putamen enlargement may be an imaging marker of responsiveness to antipsychotic treatment. Similar findings were observed in another study as well [34]. Since patients in the present study were drug-naive when they underwent the imaging assessment, the activity in the left ventromedial putamen measured in our study might be an illness-related biomarker to predict therapeutic response.

The ventromedial putamen communicates with the ventromedial thalamus, frontal cortex (the cortico-striato-thalamo-cortical circuit), and insula through multi-fiber pathways [7]. The ventromedial 

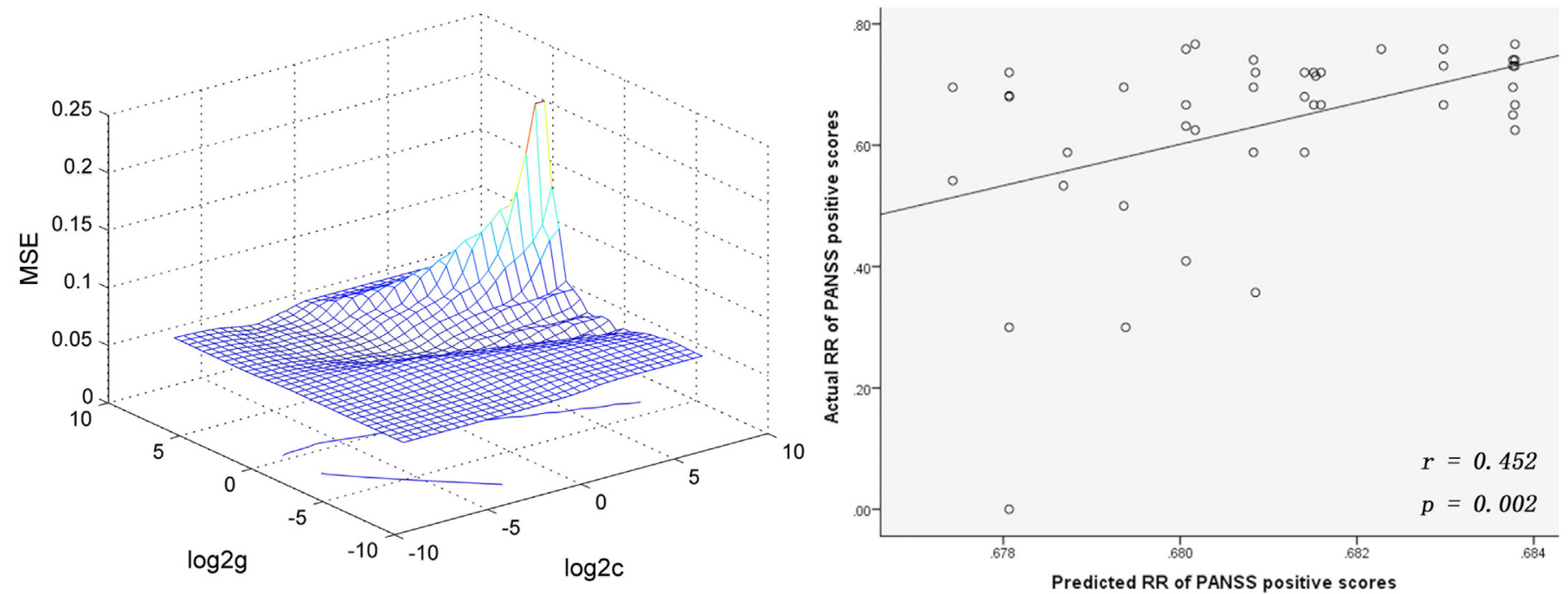

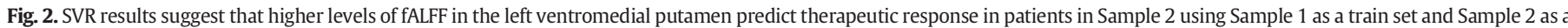

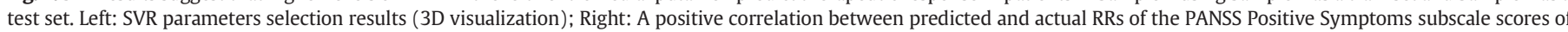

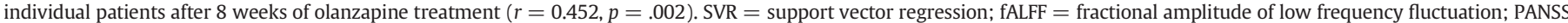
$=$ the Positive and Negative Syndrome Scale; RR = reduction rate.

putamen plays a central role in the integration and modulation of many motor, higher-level cognitive, and limbic processes [3]. Furthermore, the ventromedial putamen is rich in dopamine, a main neurotransmitter in complex behavioral and cognitive symptoms [35]. According to the dopamine hypothesis, positive symptoms are thought to result from enhanced dopamine in the subcortical regions, such as the ventromedial putamen [36]. Consistent with the dopamine hypothesis, we found increased pre-treatment activity in the left ventromedial putamen in patients in each of the two study samples, which positively correlated with the RR of the PANSS Positive Symptoms subscale scores after 8 weeks of olanzapine treatment. The left ventromedial putamen could potentially be a treatment target in future drug development.

Significant correlations were observed for AALFF values between the left and right ventromedial putamen. However, only the left ventromedial putamen, not the right ventromedial putamen, showed predictive value for positive symptom improvement after 8 weeks of olanzapine treatment. In our study, patients in both study samples showed elevated pre-treatment levels of fALFF in the left ventromedial putamen, but not in the right ventromedial putamen. As hypothesized by the data-driven method, the fact that no significant elevation of fALFF in the right ventromedial putamen in the patients of Sample 2 might prohibit its predictive role for treatment response. We also tested the predictive value of fALFF in the left/right dorsolateral putamen using the SVR method. No significant relationships were found between pre-treatment levels of fALFF in the left/right dorsolateral putamen and week 8 RR values of the PANSS total or subscale scores with one sample as a train set and another sample as a test set.

Our findings are consistent with a previous report [23], which showed an increased left putamen volume in those with a better outcome in patients with chronic schizophrenia. In addition, our findings are in line with the results from another study that reported a correlation between a larger size in the left ventral putamen and less severe paranoid symptoms in individuals with schizotypal personality disorder, and individuals with a larger size in the left ventral putamen responded better to antipsychotic treatment [37].
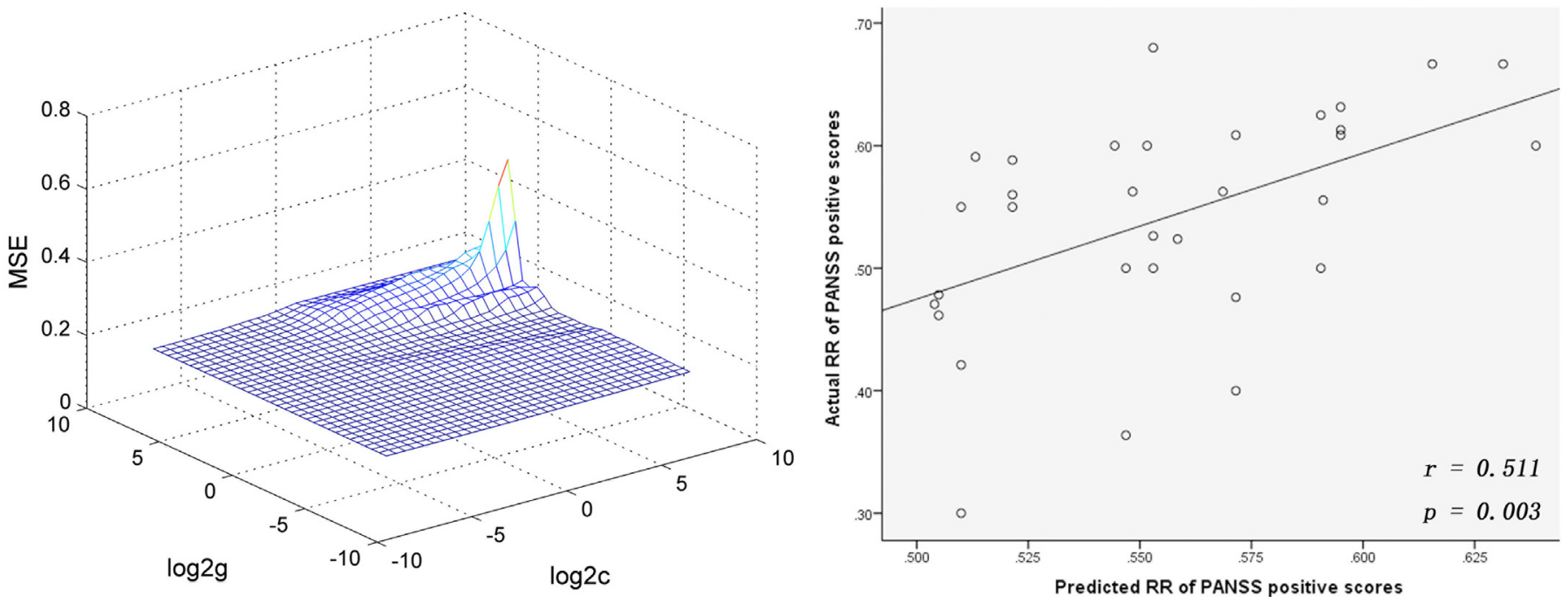

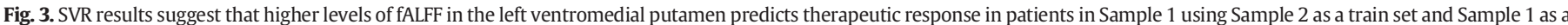

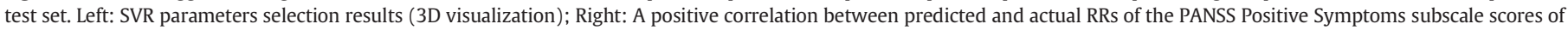

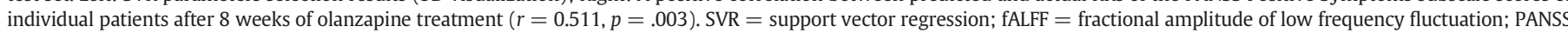
$=$ the Positive and Negative Syndrome Scale; $R R=$ reduction rate. 
There are several novel aspects in the present study. First, drugnaive, first-episode patients with schizophrenia were enrolled in each study sample, minimizing important potential confounding factors such as prior exposure to antipsychotic medications and chronicity of illness. Second, SVR was employed to predict treatment response in schizophrenia. Compared with traditional univariate analysis, pattern classification techniques can individually classify patients with good response from those with poor response. Hence, the established pretreatment predictor can help psychiatrists make an informed, individualized decision on the choice of antipsychotic medications when initiating a treatment. Third, two independent study samples were recruited in the present study. The fact that the findings were consistent across the two samples cross-validates the predictive value of the left ventromedial putamen activity for antipsychotic treatment response. Finally, a human brainnetome atlas was employed in the present study to divide putamen into four subregions: left/right ventromedial putamen and left/right dorsolateral putamen; this allows the data to be processed with fine-grained parcellations [22].

In addition to a relatively small study sample size, the present study has several limitations. First, olanzapine monotherapy was used in our study. It is helpful to minimize the confounding effect caused by different antipsychotic medications. However, it is unclear whether our findings are generalizable to patients treated with other antipsychotic medications as different medications may have differential effects on brain activity $[19,20]$. Second, it is unclear whether our findings are generalizable to chronic patients with schizophrenia who have been exposed to various antipsychotic medications, and/or have co-occurring substance use problems. Third, patients in Sample 1 and Sample 2 did not match in several demographic and clinical variables, which might limit the comparisons in outcome measures between Sample 1 and Sample 2. However, such differences may increase the generalizability of the present findings considering that schizophrenia is a highly heterogenous disease.

In conclusion, our study suggests that elevated pre-treatment mean fALFF in the left ventromedial putamen may predict better individual therapeutic response in positive symptoms to olanzapine treatment in drug-naive, first-episode patients with schizophrenia. Larger-scale future studies to further examine the clinical utility of using putamen activity as a biomarker to predict treatment response in real world patients with schizophrenia are warranted.

\section{Author contributions}

Dr. Wenbin Guo had full access to all the data in the study and takes responsibility for the integrity of the data and the accuracy of the data analysis. Study concept and design: Wenbin Guo, Xiaoduo Fan, Jingping Zhao. Acquisition, analysis, or interpretation of data: Feng Liu, Jindong Chen, Qinji Su, Zhikun Zhang, Huabing Li. Drafting of the manuscript: Huabing Li, Wenbin Guo, Xiaoduo Fan. Critical revision of the manuscript for important intellectual content: Wenbin Guo, Xiaoduo Fan, Feng Liu. Statistical analysis: Wenbin Guo, Feng Liu. Obtained funding: Wenbin Guo, Jingping Zhao. Administrative, technical, or material support: Wenbin Guo, Feng Liu, Jindong Chen, Qinji Su, Zhikun Zhang, Huabing Li, Jingping Zhao. Study supervision: Wenbin Guo, Xiaoduo Fan, Jingping Zhao.

\section{Funding/support}

This study was supported by grants from the National Key R\&D Program of China (2016YFC1307100 and 2016YFC1306900) and the National Natural Science Foundation of China (Grant Nos. 81571310, 81771447, and 81630033).

\section{Role of the funder/sponsor}

The funding sources had no role in the design and conduct of the study; collection, management, analysis, and interpretation of the data; preparation, review, or approval of the manuscript; and decision to submit the manuscript for publication.

\section{Declaration of Competing Interest}

Dr. Fan reported receiving research support from the National Institute on Alcohol Abuse and Alcoholism, National Institute on Drug Abuse, the Stanley Medical Research Institute, the Baer Foundation, the Shine Foundation, the Vanguard Group, Janssen, Avanir Pharmaceuticals, Neurocrine, and Alkermes; and reported receiving honoraria for serving on an advisory board for Allergen. Other authors reported no disclosures.

\section{Appendix A. Supplementary data}

Supplementary data to this article can be found online at https://doi. org/10.1016/j.ebiom.2019.07.022.

\section{References}

[1] Haber SN. The primate basal ganglia: parallel and integrative networks. J Chem Neuroanat 2003;26(4):317-30.

[2] Simpson EH, Kellendonk C, Kandel E. A possible role for the striatum in the pathogenesis of the cognitive symptoms of schizophrenia. Neuron 2010;65(5):585-96.

[3] Bernacer J, Prensa L, Gimenez-Amaya JM. Distribution of GABAergic interneurons and dopaminergic cells in the functional territories of the human striatum. PLoS One 2012;7(1):e30504

[4] Obeso JA, Rodriguez-Oroz MC, Stamelou M, Bhatia KP, Burn DJ. The expanding universe of disorders of the basal ganglia. Lancet 2014;384(9942):523-31.

[5] Howes OD, Montgomery AJ, Asselin MC, Murray RM, Grasby PM, McGuire PK. Molecular imaging studies of the striatal dopaminergic system in psychosis and predictions for the prodromal phase of psychosis. Br J Psychiatry Suppl 2007;51:s13-8.

[6] Luo N, Sui J, Chen J, et al. A schizophrenia-related genetic-brain-cognition pathway revealed in a large Chinese population. EBioMedicine 2018;37:471-82.

[7] Li M, Chen Z, Deng W, et al. Volume increases in putamen associated with positive symptom reduction in previously drug-naive schizophrenia after 6 weeks antipsychotic treatment. Psychol Med 2012:42(7):1475-83.

[8] McCarley RW, Wible CG, Frumin M, et al. MRI anatomy of schizophrenia. Biol Psychiatry 1999;45(9):1099-119.

[9] Boonstra G, van Haren NE, Schnack HG, et al. Brain volume changes after withdrawal of atypical antipsychotics in patients with first-episode schizophrenia. J Clin Psychopharmacol 2011;31(2):146-53.

[10] Prince JA, Harro J, Blennow K, Gottfries CG, Oreland L. Putamen mitochondrial energy metabolism is highly correlated to emotional and intellectual impairment in schizophrenics. Neuropsychopharmacology 2000;22(3):284-92.

[11] Stip E, Mancini-Marie A, Fahim C, Bentaleb LA, Letourneau G, Potvin S. Decrease in basal ganglia grey matter density associated with atypical antipsychotic treatment in schizophrenia patients. Schizophr Res 2008;103(1-3):319-21.

[12] Glenthoj A, Glenthoj BY, Mackeprang T, et al. Basal ganglia volumes in drug-naive first-episode schizophrenia patients before and after short-term treatment with either a typical or an atypical antipsychotic drug. Psychiatry Res 2007;154(3): 199-208.

[13] Guo W, Liu F, Liu J, et al. Abnormal causal connectivity by structural deficits in firstepisode, drug-naive schizophrenia at rest. Schizophr Bull 2015;41:57-65.

[14] Perkins DO, Gu H, Boteva K, Lieberman JA. Relationship between duration of untreated psychosis and outcome in first-episode schizophrenia: a critical review and meta-analysis. Am J Psychiatry 2005;162(10):1785-804.

[15] Marshall M, Lewis S, Lockwood A, Drake R, Jones P, Croudace T. Association between duration of untreated psychosis and outcome in cohorts of first-episode patients: a systematic review. Arch Gen Psychiatry 2005;62(9):975-83.

[16] Guo W, Liu F, Chen J, et al. Olanzapine modulation of long- and short-range functional connectivity in the resting brain in a sample of patients with schizophrenia. Eur Neuropsychopharmacol 2017;27:48-58.

[17] Guo W, Liu F, Chen J, et al. Olanzapine modulates the default-mode network homogeneity in recurrent drug-free schizophrenia at rest. Aust N Z J Psychiatry 2017;51 (10):1000-9.

[18] Guo W, Liu F, Chen J, et al. Treatment effects of olanzapine on homotopic connectivity in drug-free schizophrenia at rest. World J Biol Psychiatry 2018;19(sup3) (S106S14).

[19] Lahti AC, Holcomb HH, Weiler MA, Medoff DR, Tamminga CA. Functional effects of antipsychotic drugs: comparing clozapine with haloperidol. Biol Psychiatry 2003; 53(7):601-8.

[20] Molina V, Gispert JD, Reig S, et al. Cerebral metabolism and risperidone treatment in schizophrenia. Schizophr Res 2003;60(1):1-7.

[21] Hutcheson NL, Clark DG, Bolding MS, White DM, Lahti AC. Basal ganglia volume in unmedicated patients with schizophrenia is associated with treatment response to antipsychotic medication. Psychiatry Res 2014;221(1):6-12.

[22] Fan L, Li H, Zhuo J, et al. The human brainnetome atlas: a new brain atlas based on connectional architecture. Cereb Cortex 2016;26(8):3508-26. 
[23] Mitelman SA, Canfield EL, Chu KW, et al. Poor outcome in chronic schizophrenia is associated with progressive loss of volume of the putamen. Schizophr Res 2009; 113(2-3):241-5.

[24] Gao S, Calhoun VD, Sui J. Machine learning in major depression: from classification to treatment outcome prediction. CNS Neurosci Ther 2018;24(11):1037-52.

[25] Osuch E, Gao S, Wammes M, et al. Complexity in mood disorder diagnosis: fMRI connectivity networks predicted medication-class of response in complex patients. Acta Psychiatr Scand 2018;138(5):472-82.

[26] Wang S, Zhan Y, Zhang Y, et al. Abnormal long- and short-range functional connectivity in adolescent-onset schizophrenia patients: a resting-state fMRI study. Prog Neuropsychopharmacol Biol Psychiatry 2018;81:445-51.

[27] Liu Y, Zhang Y, Lv L, Wu R, Zhao J, Guo W. Abnormal neural activity as a potential biomarker for drug-naive first-episode adolescent-onset schizophrenia with coherence regional homogeneity and support vector machine analyses. Schizophr Res 2018;192:408-15

[28] Wang S, Zhang Y, Lv L, et al. Abnormal regional homogeneity as a potential imaging biomarker for adolescent-onset schizophrenia: a resting-state fMRI study and support vector machine analysis. Schizophr Res 2018;192:179-84.

[29] Redlich R, Opel N, Grotegerd D, et al. Prediction of individual response to electroconvulsive therapy via machine learning on structural magnetic resonance imaging data. JAMA Psychiat 2016;73(6):557-64.
[30] First MB, Spitzer RL, Gibbon M, Williams JBW. Structured Clinical Interview for DSMIV Axis I Disorders (SCID). Washington, DC: American Psychiatric Press; 1997.

[31] Yan CG, Wang XD, Zuo XN, Zang YF. DPABI: data processing \& analysis for (restingstate) brain imaging. Neuroinformatics 2016;14(3):339-51.

[32] Zou QH, Zhu CZ, Yang Y, et al. An improved approach to detection of amplitude of low-frequency fluctuation (ALFF) for resting-state fMRI: fractional ALFF. J Neurosci Methods 2008;172(1):137-41.

[33] Power JD, Barnes KA, Snyder AZ, Schlaggar BL, Petersen SE. Spurious but systematic correlations in functional connectivity MRI networks arise from subject motion. Neuroimage 2012;59:2142-54.

[34] Buchsbaum MS, Shihabuddin L, Brickman AM, et al. Caudate and putamen volumes in good and poor outcome patients with schizophrenia. Schizophr Res 2003;64(1): 53-62.

[35] Schultz W. Behavioral dopamine signals. Trends Neurosci 2007;30(5):203-10.

[36] Howes OD, Kapur S. The dopamine hypothesis of schizophrenia: version III-the final common pathway. Schizophr Bull 2009;35(3):549-62.

[37] Chemerinski E, Byne W, Kolaitis JC, et al. Larger putamen size in antipsychotic-naive individuals with schizotypal personality disorder. Schizophr Res 2013;143(1): 158-64. 